\title{
Cystoid macular edema secondary to paclitaxel therapy for ovarian cancer: A case report
}

\author{
EMMA BASSI $^{1}$, VERA LOIZZI ${ }^{1,2}$, CLAUDIO FURINO $^{3}$, ROSA MARTINO $^{3}$, \\ GIOVANNI ALESSIO ${ }^{3}$, CICINELLI ETTORE ${ }^{1}$ and GENNARO CORMIO ${ }^{1,2}$ \\ ${ }^{1}$ Department of Biomedical Science and Human Oncology, Obstetrics and Gynecology Unit, University of Bari; \\ ${ }^{2}$ IRCCS ‘Giovanni Paolo II'; ${ }^{3}$ Department of Ophthalmology, University of Bari, I-70124 Bari, Italy
}

Received September 14, 2016; Accepted March 13, 2017

DOI: $10.3892 / \operatorname{mco} .2017 .1296$

\begin{abstract}
Paclitaxel is a member of the taxane agents that has demonstrated efficacy in ovarian cancer, both in first- and in second-line therapy. Counted among the side-effects of this drug are neurological disorders. In the present study, a rare case of a non-neuropathic ocular disorder, known as cystoid macular edema (CME), due to paclitaxel in patients treated for ovarian cancer is described. Macular edema, or CME, is a common cause of visual impairment that has been classically demonstrated by fluorescein angiograms, showing capillary leakage. CME without fluorescein leakage is rare, and its most common causes are juvenile X-linked retinoschisis, Goldmann-Favre syndrome, and niacin toxicity. At the present time, the mechanism associated with the form of CME that does not exhibit any signs of fluorescein leakage has not been elucidated due to an absence of histopathological studies. Several mechanisms have been proposed, although it is considered to occur due to disruption of the normal blood-retinal barrier by molecules with a molecular weight lower than that of fluorescein, which leads to fluid accumulation in the intracellular space. It is well known that taxane agents cause fluid retention, represented by edema, weight gain, and third-space fluid collection (pericardial, pleural, ascites), and this appears to be associated with their cumulative dose. The present case study confirms that macular edema associated with paclitaxel use exhibits spontaneous resolution following discontinuation of the causative agent. Taxane-associated maculopathy has been scarcely reported in the literature, but the gynecological oncologist should be alert to its possible development, and an ophthalmologic evaluation should be offered to all patients using paclitaxel.
\end{abstract}

Correspondence to: Professor Vera Loizzi, Department of Biomedical Science and Human Oncology, Obstetrics and Gynecology Unit, University of Bari, Piazza Giulio Cesare 11, I-70124 Bari, Italy

E-mail: vloizzi@tiscali.it

Key words: chemotherapy, ovarian cancer, taxanes

\section{Introduction}

Paclitaxel is a member of the taxane agents that has demonstrated efficacy in ovarian cancer, both in first- and in second-line therapy (1). Neurological disorders are counted among the side-effects of this drug (2). In the present study, a rare case of a non-neuropathic ocular disorder known as cystoid macular edema (CME), due to the use of paclitaxel in patients treated for ovarian cancer, is described.

\section{Case report}

In September 2007, a 49-year-old woman underwent optimal debulking surgery for FIGO stage IIIC ovarian serious cancer, with residual disease following surgery (a tumor measuring $<1 \mathrm{~cm}$ ). The patient received adjuvant chemotherapy based on carboplatin and paclitaxel for six cycles every three weeks, achieving a complete response. The cumulative dose of paclitaxel was $1,680 \mathrm{mg}$. After 13 months, the patient presented with hepatic metastasis and peritoneal nodules. In view of the recurrence of the disease, the patient received chemotherapy based on carboplatin and pegylated liposomal doxorubicin. After 8 cycles of the chemotherapy, based on the disease progression the patient subsequently received Topotecan for 13 cycles. However, the patient then presented with pulmonary progression disease, and consequently she was treated with paclitaxel $\left(60 \mathrm{mg} / \mathrm{m}^{2}\right)$ administered intravenously once a week. At the 45 th cycle of chemotherapy, the patient was referred to the ophthalmologist for progressively decreasing bilateral visual acuity. The visual acuity was 20/200 in the right eye (RE) and 20/100 in the left eye (LE), and it did not improve through the use of lenses. The slit lamp biomicroscopy of the anterior segment and the intraocular pressure of both eyes were normal, but fundus ophthalmoscopy revealed a bilateral CME. Spectral domain optical coherence tomography (OCT) (Cirrus OCT; Zeiss GmbH, Jena, Germany) scans of the two eyes revealed an increased macular thickness due to intraretinal fluid accumulation; fluorescein angiography exhibited normal filling of the choroidal and retinal vessel, with an intact parafoveal capillary net. These findings were consistent with the diagnosis of CME without leaking vessels. The patient's optical history was negative for previous eye diseases. After collegial counseling based on paclitaxel side-effects, the 

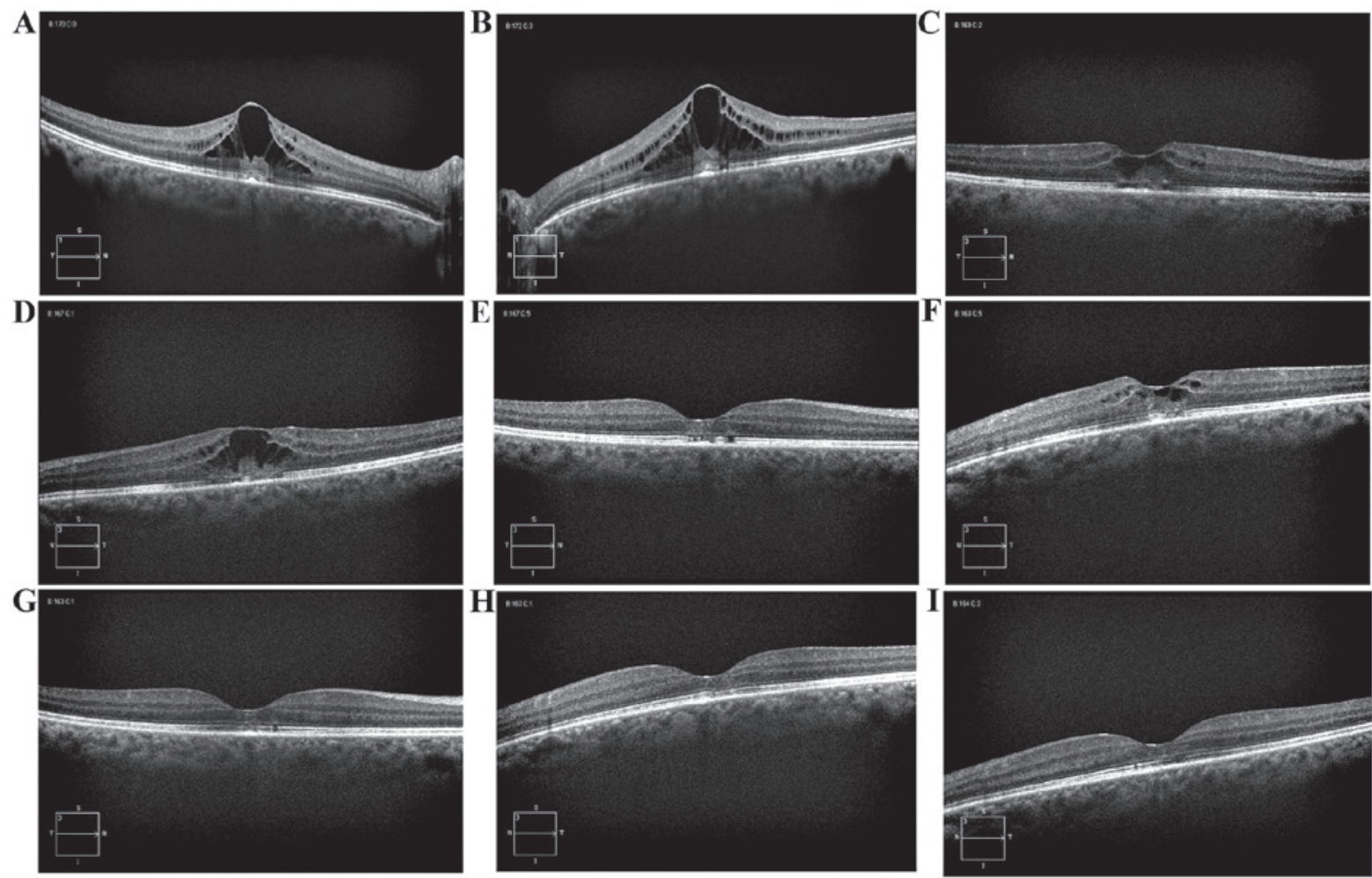

Figure 1. Spectral domain optical coherence tomography images derived from the present case study. (A) Baseline: Macular edema with a CRT of $515 \mu \mathrm{m}$ in the RE. (B) Baseline: Macular edema with a CRT of $584 \mu \mathrm{m}$ in the LE. (C) At 40 days after the interruption of plaxitel systemic therapy, CRT decreased to $320 \mu \mathrm{m}$ in the RE. (D) At 40 days after the interruption of plaxitel systemic therapy, CRT decreased to $391 \mu \mathrm{m}$ in the LE. (E) Three months later, the CRT was $232 \mu \mathrm{m}$ in the RE, with the restoration of macular shape. (F) Three months later, the CRT was $316 \mu \mathrm{m}$ in the LE, with the restoration of macular shape. (G) Four months later, the CRT was $230 \mu \mathrm{m}$ in the RE. (H) Four months later, the CRT was $227 \mu \mathrm{m}$ in the LE. (I) Eight months later, the CRT was $235 \mu \mathrm{m}$ in the RE, with a normal macula shape. (J) Eight months later, the CRT was 234 microns in the LE, with a normal macula shape. CRT, central retinal thickness; LE, left eye; RE, right eye.

chemotherapy treatment was discontinued. Thirty days later, the visual acuity increased (20/50 in the RE and 20/40 in the LE). Three months after the discontinuation of paclitaxel, visual acuity increased to 20/20 in both eyes, and a further OCT examination revealed the restoration of macular shape, with regression of macular edema. The patient remains alive with stable disease, and without treatment (Fig. 1).

\section{Discussion}

Paclitaxel is an anticancer drug produced by the plants of the genus Taxus, which acts by restricting microtubule mobility and inhibiting mitosis. The largest and heaviest adverse effect of this reagent is toxic effects to the bone marrow and neurological disorders. The frequency of peripheral neuropathy has been reported to increase with a cumulative dose. Reports concerning ophthalmic adverse effects, including impaired visual acuity, dry eyes, scintillating scotomas, keratitis, photopsia, development of open-angle glaucoma and optic neuropathy, are scarce $(3,4)$. Furthermore, bilateral CME has been described as a highly unusual adverse event after the administration of taxanes. Macular edema, or CME, is a common cause of visual impairment, and has been classically demonstrated using fluorescein angiograms, showing capillary leakage. CME without fluorescein leakage occurs only rarely, and its most common causes are juvenile $\mathrm{X}$-linked retinoschisis, Goldmann-Favre syndrome, and niacin toxicity $(3,5,6)$. At the present time, the mechanism of CME associated with no fluorescein leakage has not been elucidated, since the necessary histopathological studies have not been performed. Several mechanisms have been proposed, although it is considered to result from disruption of the normal bloodretinal barrier by molecules having a molecular weight lower than that of fluorescein. This leads to fluid accumulation in the intracellular space. It has been well established that taxane agents cause fluid retention, represented by edema, weight gain, and third-space fluid collection (pericardial, pleural, ascites), and this appears to be associated with their cumulative dose $(7,8)$. Considering the cases where there was no indication of systemic fluid retention, Koo and Kim (9) have suggested that CME was presumed to occur as a result of cellular toxicity derived from the suppression of intracellular microtubule reorganization (9).

It has yet to be determined why, even assuming a specific pathogenetic mechanism, this side-effect occurs so infrequently. In the literature, there are 13 cases of CME associated with the use of taxanes, including 6 caused by Taxol, 5 due to nanoparticle albumin-bound paclitaxel, and only 2 linked with Taxotere (docetaxel) (3,10-17). It remains an open question as to why paclitaxel is more toxic than docetaxel. Possibly, a certain amount of cumulative dose is necessary to induce CME, and this may be achieved less easily through the use of docetaxel. To the best of our knowledge, the present case study is the first that has been reported in the field of ovarian 
cancer, since those previously described in literature occurred during the course of treatment of lung or breast cancer. The second peculiarity of the present case report lies in the fact that the toxicity was presented only during the use of Taxol in the second line of chemotherapy, and only when it had reached a very high cumulative dose, such as 4,380 mg.

Considering the very late manifestation reported in the present case study, it is possible to envisage a sensitizing effect, although further studies are required in order to confirm this. Based on the literature, it is evident that the treatment of CME has not uniquely been based upon paclitaxel therapy: Cases have also been described of the use of acetazolamide, prednisolone, triamcinolone and dorzolamide. However, to the best of our knowledge, the present case study has confirmed that macular edema associated with paclitaxel use exhibited spontaneous resolution following discontinuation of the causative agent. Taxane-associated maculopathy has been scarcely reported in the literature, but the gynecological oncologist should be alert to its possible development, and an ophthalmologic evaluation should be offered to all patients using paclitaxel.

\section{References}

1. Boere IA and van der Burg ME: Review of dose-intense platinum and/or paclitaxel containing chemotherapy in advanced and recurrent epithelial ovarian cancer. Curr Pharm Des 18: 3741-3753, 2012.

2. Oishi R and Egashira N: Fukuoka. Peripheral neuropathy induced by anticancer drugs. IgakuZasshi 104: 71-80, 2013.

3. Hofstra LS, de Vries EG and Willemse PH: Ophthalmic toxicity following paclitaxel infusion. Ann Oncol 8: 1053, 1997.

4. Li J, Tripathi RC and Tripathi BJ: Drug-induced ocular disorders. Drug Saf 31: 127-141, 2008.
5. Semb KA, Aamdal S and Oian P: Capillary protein leak syndrome appears to explain fluid retention in cancer patients who receive docetaxel treatment. J Clin Oncol 16: 3426-3432, 1998.

6. Spirn MJ, Warren FA, Guyer DR, Klancnik JM Jr and Spaide RF Optical coherence tomography findings in nicotinic acid maculopathy. Am J Ophthalmol 135: 913-914, 2003.

7. Telander DG and Sarraf D: Cystoid macular edema with docetaxel chemotherapy and the fluid retention syndrome. Semin Ophthalmol 22: 151-153, 2007.

8. Michaud LB, Valero V and Hortobagyi G: Risks and benefits of taxanes in breast and ovarian cancer. Drug Saf 23: 401-428, 2000.

9. Béhar A, Pujade-Lauraine E, Maurel A, Brun MD, Chauvin FF, Feuilhade de Chauvin F, Oulid-Aissa D and Hille D: The pathophysiological mechanism of fluid retention in advanced cancer patients treated with docetaxel, but not receiving corticosteroid comedication. Br J Clin Pharmacol 43: 653-658, 1997.

10. Koo NK and Kim YC: A case of paclitaxel-induced maculopathy treated with methazolamide. Korean J Ophthalmol 26: 394-397, 2012.

11. Teitelbaum BA and Tresley DJ: Cystic maculopathy with normal capillary permeability secondary to docetaxel. Optom Vis Sci 80: 277-279, 2003

12. Murphy CG, Walsh JB, Hudis CA, Lake D and Theodoulou M: Cystoid macular edema secondary to nab-paclitaxel therapy. J Clin Oncol 28: e684-e687, 2010.

13. Smith SV, Benz MS and Brown DM: Cystoid macular edema secondary to albumin-bound paclitaxel therapy. Arch Ophthalmol 126: 1605-1606, 2008.

14. Joshi MM and Garretson BR: Paclitaxel maculopathy. Arch Ophthalmol 125: 709-710, 2007.

15. Risard SM, Pieramici DJ and Rabena MD: Cystoid macular edema secondary to Paclitaxel (abraxane). Retin Cases Brief Rep 3: 383-385, 2009.

16. Georgakopoulos CD, Makri OE, Vasilakis P and Exarchou A: Angiographically silent cystoid macular oedema secondary to paclitaxel therapy. Clin Exp Optom 95: 233-236, 2012.

17. Baskin DE and Garg SJ: Abraxane-induced cystoid macular edema refractory to concomitant intravenous bevacizumab. Can J Ophthalmol 46: 200-201, 2011. 